\title{
Mondialisation, travail social et politique du sujet
}

\section{Mundialização, trabalho social e política do sujeito}

\author{
Mundialization, social work and subject's policy
}

Jean BIARNÈS

\begin{abstract}
RESUME $^{1}$
L'article présente quelques possibilités d'une politique du sujet, il englobe les questions d'éthique et de démocratie. Le point de départ est l'analyse du travail réalisé par deux groupes du Programme EQUAL. Ces groupes tiennent compte de l'insertion sociale des jeunes en France. Selon l'auteur, cette analyse devrait prendre en même temps compte de la dimension quantitative et de la dimension qualitative du point de vue de la personne comme individu. De cette manière, il apparaît comme conséquence urgente que les acteurs sociaux s'inquiètent à construire leurs propres instruments d'évaluation de leur travail, instruments d'évaluation qualitative et non quantitative, en se respectant une vraie dynamique du sujet, tant du côté de l'utilisateur que de l'acteur social dans lequel chacun à la nécessité " se démasquer ", d'expliciter leurs critères sous le regard des situations vives en commun et d'essayer de rester cohérent. Tout ce travail doit prendre en compte que « être je soumets » ne peut pas jamais être isolé de l'objet. L'« être sujet » est un « être dans une situation ». « Être sujet » est être un " sujet qui parle et qui agit », c'est-à-dire, un " sujet qui désire », dans un scénario social où le domaine mondial est délimité par les frontières inexactes à l'intérieur de leurs concrétisations et d'une extrême efficacité à l'intérieur de leurs fonctionnements. Dans ce contexte, instruire - former, accompagner la dimension du désir créatif du sujet - est la première mission qui devrait être donné à tout travailleur social, par lui-même et par son travail avec l'autre. Cette connaissance du sujet et de son propre mot implique que l'acteur social « fasse avec » le sujet et non « pour » le sujet.
\end{abstract}

Mots clés : acteur social, insertion sociale, mondialisation, sujet, travail social.

\footnotetext{
${ }^{1}$ Traduzido para o francês por: Eliana Mendes de Moraes que cursa o $5^{\circ}$ semestre de psicologia na Universidade Paulistana e trabalha na Fundação Liceu Pasteur; 2008.
} 


\title{
RESUMO
}

O artigo apresenta algumas possibilidades de uma política do sujeito, abrangendo as questões da ética e da democracia, partir da análise do trabalho realizado em dois grupos do Programa EQUAL. Esses grupos acompanham a inserção social de jovens na França. Para o autor, eles deveriam avaliar-se levando em consideração, ao mesmo tempo, a dimensão quantitativa e a dimensão qualitativa em relação à pessoa enquanto sujeito. Desse modo, surge como conseqüência urgente que os atores sociais se preocupem em construir seus próprios instrumentos de avaliação do seu trabalho, instrumentos de avaliação qualitativa e não quantitativa, respeitando-se uma verdadeira dinâmica do sujeito, tanto do lado do usuário como do ator social na qual cada um tem a necessidade de se "desvendar" (biografização recíproca), de explicitar seus critérios sob o olhar das situações vividas em comum (re-territorialização da pessoa) e de tentar permanecer coerente (desparcialização da pessoa dentro dos diferentes percursos). Todo esse trabalho deve levar em conta que "ser sujeito" não pode jamais ser isolado do objeto. O "ser sujeito" é um "ser em situação”. "Ser sujeito" é ser um "sujeito que fala e que age”, ou seja, um "sujeito que deseja”, num cenário social em que o domínio mundial é demarcado pelas fronteiras imprecisas dentro de suas concretizações e de uma extrema eficácia dentro dos seus funcionamentos. Nesse contexto, educar - formar, acompanhar a dimensão do desejo criativo do sujeito - é a primeira missão que deveria ser dada a todo trabalhador social, por ele mesmo e pelo seu trabalho com o Outro. Este conhecimento do sujeito e da sua própria palavra implica que o ator social "faça com” o sujeito e não "para” o sujeito.

Palavras-chave: ator social, inserção social, mundialização, sujeito, trabalho social.

\begin{abstract}
The article presents some possibilities for a subject's policy envolving question of ethics and democracy. It is based on the analysis of a work realized by two groups of the EQUAL Program. These groups accompany the social insertion of young people in France. According to the author, they should evaluate their social work taking into account, at the same time, the quantitative dimension and the qualitative dimension in relation to the person as subject. This way, it comes to sight an urgent consequence that social actors get worry about constructing their own
\end{abstract}


evaluator's instruments of their work, in order to a qualitative and no quantitative evaluate, respecting a real subject's dynamic, from the user side as well from the social actor side in which each one has the necessity of unveiling (reciprocal "biographization"), explicating their criterions according to their point of view about common lived situations (re"territorialization" of the person) and trying to permanence coherent ("desparcialization" of the person inside different trajectories). All these work ought to take into account that "being subject" can not ever be isolated from the object. The "being subject" is a "being in situation". "Being subject" is to be a "subject who talks and acts", it means, a "subject who desires", in a social scenario in which world-wide dominance is delimited by imprecisely borders inside their concretizations and an extreme efficacy inside their functioning. In this context, to educate - to form, to accompany the dimension of subject's creative desire - is the first mission that should be given to all social worker, by himself and by his work with the Other. This knowledge of the subject and his own word implicates that social actor "works with" instead of "works for" the subject.

Index terms: social actor, social insertion, mundialization, subject, social work.

\section{Qu'est-ce qu'«être sujet» veut dire aujourd'hui ?}

Si j'essaie de définir qui je suis à un moment donné, je dois être conscient qu'à l'instant suivant je ne suis plus totalement celui que j'ai défini, car, même de manière infinitésimale, le fait de me définir a changé ma propre perception de qui je suis. Le «je suis» est constitué d’un noyau de permanence et d'un état de transformation perpétuelle. Définir l’être-sujet, c'est donc l'inclure dans cette «permanence/labilité». L'être est toujours à créer. Penser réduire cette création de l'être-sujet à l'apprentissage d’actes imposés et à reproduire, c’est la nier voire la tuer. Quand une société a cet objectif, elle y parvient plus sûrement quand elle s’y prend tôt dans la vie de l'enfant, quand elle lui impose d'apprendre à reproduire des actes préétablis et des pensées toutes faites plutôt que de développer ses capacités 
créatrices ${ }^{2}$. C'est cette marge de création en acte qui permet à l'être humain de n’être pas totalement prédéterminé socialement et qui signe pour nous l'«être sujet».

Le concept de l'«être-sujet» est alors marqué du sceau de l'«action perpétuelle de sa propre création» et «d'une action perpétuelle sur le monde», action signée du lieu même de la personne à l’intérieur du cadre socialement et culturellement défini dans lequel elle se trouve. L’activité de création du «Je-sujet» lierait donc étroitement deux niveaux d'action et d'objectif : le premier niveau serait l'action, d'ordre interne, du sujet sur luimême travaillant à créer des liens entre ses appartenances à l'universel, au culturel, à différentes sub-cultures, et son être singulier; le second niveau serait l'action conduite par le sujet sur ses environnements et la manière dont il crée et inscrit son espace et son histoire individuels dans l'espace et l'histoire collectifs. «Être sujet» est donc un processus, celui de l'intégration toujours à faire et jamais achevée du « Je » à la communauté humaine et celui de la «transformation-maintien-de-la-permanence» du «Je» par cette intégration des modèles symboliques portés par cette communauté. Écrire son histoire, c’est avoir la possibilité de travailler ce processus et de créer de façon toujours plus autonome,, les liens entre tous ces éléments internes et externes.

Ce processus ne peut fonctionner que si cette action menée conjointement sur soi-même et sur ses environnements est pourvue de sens. Il est, par exemple, plus facile de travailler avec des jeunes primo-arrivants qu'avec des jeunes ayant subi des échecs scolaires et l'exclusion sociale. La violence, lorsqu'elle existe chez les premiers, a un sens précis de revendication ou d'action contre les difficultés d'intégration sociale, alors

\footnotetext{
${ }^{2}$ Or que faisons-nous aujourd'hui à l'école maternelle où le terme «travail» a supplanté le jeu, où les enfants de moyennes sections passent des «examens» pour accéder à la grande section, où tous les enfants ont des «livrets scolaires» présentant un nombre impressionnant d'items? Cf. la thèse en cours de Stéphanie Pheng, Experice Paris13.
} 
que chez les seconds la violence n'exprime qu'une lutte contre le «nonsens» ${ }^{3}$.

«Être sujet» est un processus activé dès la naissance. Le premier «problème» de l'enfant est d'apprendre à s'exclure du corps de la mère pour advenir à ce qu'il est, en tant que créateur de sa propre histoire. Le sujet est toujours dans cette déchirure. À chaque pas de sa vie, il va devoir s'exclure du «passé-présent» qu'il connaît, qui l'assure de son identité et donc le rassure, pour entrer dans l'étrangéité de l’Autre, le «présent-futur» qui va transformer son identité et donc le «dé-assure». Tout travail éducatif et social projette le sujet au cœur même de ce mouvement à double sens « insertion/désinsertion ». S’insérer dans un espace nouveau (la crèche, l'école, le monde du travail) nécessite ainsi de perdre quelque chose du monde d'avant. Être sujet, c'est pouvoir au présent assumer cette perte d’une partie du passé, face au gain espéré du futur. Les politiques sociales, en opposant «insertion» et «exclusion» font un contresens. L'insertion n'est qu'une face de ce mécanisme vital à deux faces «insertion/désinsertion» intrinsèquement liées comme un «Janus bi-frons» et qui signent «l'êtresujet».

Enfin «l'être sujet» ne peut jamais être totalement isolé de l'objet. «L'être sujet» est un «être en situation». Cette dynamique situationnelle fonde la question du désir. Faire ceci plutôt que cela, lorsque le sujet n’est pas assujetti à une contrainte totalitaire, relève du désir. Une contrainte

\footnotetext{
3 Lors d'une recherche en 1995 avec des enfants de 10 ans d'une banlieue sud de Paris nous avions mis en évidence l'état de dépression profonde de ceux-ci et nous écrivions (BIARNÈS, 1999, p.177): «Si des enfants de 10 ans sont déjà dans la dépression et que l'école ne voit ni n'entend cette «différence », alors il ne faut pas s'étonner de la violence qui se manifeste au collège. De plus, ce ne peut être que la pire des violences, celle qui n'est même plus revendicative, qui n'est même plus un appel au secours, celle qui n'a qu'un seul objet, lutter contre le néant de l'être ». En novembre 2005, ces enfants étaient devenus des jeunes de 20 ans! Comment interpréter autrement le fait qu'ils brûlèrent les voitures de personnes qu'ils côtoyaient quotidiennement, si ce n'est par ce «non-sens » de l'être ? Comment comprendre qu'ils brûlèrent aussi des écoles alors qu'en 1995, les jeunes incarcérés à la FEBEM (maison de correction/prison) à Saõ Paolo brûlèrent tous les bâtiments de cette institution/prison sauf...l'école?
} 
totalitaire serait celle d'un sujet totalement lié à l'objet ou au contraire totalement délié de l’objet. Hormis des cas pathologiques, il existe donc toujours une faille, un manque entre le sujet et l'objet dans une situation donnée, ce qui fait que le sujet est toujours un sujet incomplet en désir du monde. Être sujet, si c’est être «sujet parlant et sujet actant», c’est aussi être «sujet désirant».

\section{La mondialisation post-moderne: sociétés en «îlots» et règles de tri et de contrôle}

La «mondialisation» des rapports sociaux ne date pas d'aujourd'hui (MONGIN, 2005). Si les découvertes des deux derniers siècles en ont transformé la forme à partir, entre autres, de la révolution du «temps vécu» par la révolution des moyens de transport ou celle de l'information (HABERMAS, 1987), avant même les débuts de notre histoire moderne, les routes du sel ou de la soie, les grands mouvements d'invasion et de migration, les croisades ont mis en relation les peuples du monde.

Sur ces liens de mondialisation préexistants, l'époque des «grandes découvertes» a introduit une autre dimension, celle de la «hiérarchie» entre les mondes. L'Europe d'alors se donne un rôle de leadership et regarde les cultures autres comme n'ayant pas atteint son propre niveau de «civilisation». Pendant près de cinq siècles, elle put ainsi se fixer des missions de «civilisation» et «d'émancipation» de ces «sous-cultures», ce qui lui permit de masquer ses objectifs d'exploitation des hommes et des ressources.

Si, dans cette longue histoire de la conquête et de la colonisation, les pays dominants ont pu situer les «barbares» de l'autre côté de la mer, aujourd'hui les «barbares», parfois aussi appelés «sauvageons» ou «racailles», sont de l’autre côté de la rue. La mondialisation actuelle, par la transformation du temps et de l'espace, a modifié la territorialisation de la 
dominance. Les frontières nationales ne sont plus les remparts de la domination des uns sur le reste du monde. Il y a «dénationalisation» et donc «déterritorialisation» du pouvoir par la parcellisation de celui-ci en «îlots» à l’intérieur des frontières nationales. C'est ce que Miguel Benasayag désigne sous le terme «d'individu». «L'individu est un nombre de personnes reliées par les liens de l'argent, du profit et du pouvoir»(BENASAYAG, 2004, p.27). Ces «îlots» intra-nationaux forment un réseau international du pouvoir grâce aux nouvelles technologies de l'information et de la communication. Les personnes composant ses «îlots» sont en relation perpétuelle avec leur alter ego aux antipodes mais ne parlent pas à leurs voisins de palier.

La toile qui constitue aujourd'hui la dominance mondiale se démarque par des frontières à la fois floues dans leurs concrétisations et d'une extrême efficacité dans leurs fonctionnements. Les sociétés postmodernes sont ainsi constituées de ces systèmes en «îlots» clos sur euxmêmes qui s'auto-alimentent, s'auto-reproduisent et s'auto-évaluent. Le reste est laissé à la «barbarie». Ce monde dual tue la démocratie.

En effet plus les frontières entre les «territoires» sont floues, plus elles ont besoin, pour s'assurer, d'être dans le rejet de l'Autre différent, donc de l'identifier et de le maîtriser. Il faut avoir vécu concrètement les «ghettos de riches» aux des États-Unis d'Amérique (USA) pour comprendre ce mécanisme du «communautarisme/intégrisme dont le «profit/pouvoir» est la valeur suprême. Ces îlots sont donc des machines à exclure l'autre. Mais si les frontières nationales existent encore pour exclure le «barbare» venant de l'autre côté de la mer, elles n’ont qu'une efficacité réduite pour exclure celui qui est de l'autre côté de la rue. Il faut alors se doter de «règles» de tri et de contrôle qui vont permettre d'identifier facilement le «semblable», et donc à fortiori «l'autre». Pour savoir et pouvoir placer chacun du bon côté de ces frontières floues, il est nécessaire que tout le 
système soit «limpide», «lisible» à tout moment. Apparaissent alors trois types de règles, celles de «l’ingénieur», du «médecin» et du «comptable».

Pour l'ingénieur, le réel doit être entièrement contrôlable. Or s’il est possible de contrôler le réel matériel, l’humain échappe, lui, à la possibilité d'un contrôle total. Pour faire croire qu'il y parvient, l’ingénieur en exclut la complexité par une segmentation du réel qu'il pourra contrôler au prix d'un rationalisme totalisant qui confine au scientisme. Le «compliqué» remplace la complexité (MORIN, 1992) et l’ingénieur sait alors construire toute chose par assemblage des parties qu’il a su isoler. Ce fantasme du «réelreconstruit» devient alors pour lui «le réel» qu’il impose comme tel au reste de la société. Ses titres et ses diplômes, labellisés par le nom des grandes écoles d'où il est issu, le protègent de questions qui ne pourraient être que «naïves», surtout si elles viennent de cet autre côté de la rue, de ce domaine laissé aux «barbares» ${ }^{4}$.

Pour que la transparence du système soit réelle, tous les «plis» et «replis» de la complexité humaine doivent disparaître. La pensée de l'ingénieur exclut cette chose inconvenante que l'on appelle l'imaginaire, c'est-à-dire «le non-maîtrisable», ou encore simplement «l'humain». Combien de décideurs dans les dispositifs d'action sociale définissent comme «verbiage» et «perte de temps» le discours des acteurs sociaux qui eux parlent de la personne avec laquelle ils travaillent chaque jour? La «chose humaine» est segmentée en parties analysables, et donc «délocaliséee» car la mise en situation réintroduirait la complexité.

Les règles de l’ingénieur structurées à partir des années soixante, ont été complétées à partir des années quatre-vingts par les règles du médecin. Ces règles d' «hygiène» du système passent par la voie de la médicalisation de ses dysfonctionnements possibles, en vue d'éliminer la complexité

\footnotetext{
${ }^{4}$ Ainsi l'ingénieur a pu construire des abattoirs ultramodernes à la Villette qu'il a fallu raser parce qu'il avait simplement oublié de questionner le paysan sur l'inclinaison maximale des pentes d'accès que les animaux pouvaient emprunter !
} 
existante dans toute problématique relationnelle. Si un enfant présente des symptômes de non-adaptation ou de résistance à des situations sociales et institutionnelles, cela ne peut venir que de gênes mal formés ${ }^{5}$. Les progrès de la génétique servent alors de fondement scientiste à ce type de démonstration ${ }^{6}$. Ainsi la dyslexie n'est pas un trouble de la maîtrise de la langue par lequel l'enfant qui en est affecté dit quelque chose au monde qui l'entoure, elle ne peut être qu’une maladie génétique. Pour preuve, à l'école, le dépistage de ce trouble n’est plus laissé aux pédagogues ou aux psychologues scolaires, mais est réservé au médecin scolaire, dans le cabinet où il vient de procéder à l'examen médical de l'enfant ! Et que dire de l'entreprise de «triage » des enfants de trois ans dits «hyperactifs» pour prévenir la délinquance à l’âge de l'adolescence ${ }^{7}$.

Enfin, aujourd'hui, à côté des règles du "réel contrôlé » et de «l'hygiénisme systémique», fonctionne la règle du «comptable», la règle du «tout évaluable» et de la rentabilité maximale. Les entreprises sont devenues les piliers de cette règle du comptable. L'entreprise n'est plus une communauté de personnes travaillant, chacun à son niveau, à la production de biens. Elle est devenue une machine à produire de l'argent à court terme pour alimenter des «îlots» de pouvoir. Les «actionnaires», le plus souvent à partir des fonds de pension nord-américains, n’ont qu'une obsession, les dividendes. Réinvestir dans la recherche, dans la modernisation n’a pas de sens dans cette perspective. Que dire alors d'investissements qui viseraient à

\footnotetext{
5 Margaret Mead (1935) faisait le même constat chez les Arapesh, communauté de Nouvelle Guinée, organisée sur des principes de non-violence. Si une séquence agressive se déclarait entre deux individus, cela ne pouvait venir que de maléfices envoyés par les indiens des plaines. Des rituels purificateurs chassaient alors ces maléfices hors de la communauté qui pouvait ainsi retrouver sa pureté originelle. Le scientisme génétique a aujourd'hui, dans nos communautés de dominants, la même fonction magico-religieuse.

${ }^{6}$ Nous ne nions nullement les causes biologiques dans l'étiologie de ce type de troubles, nous disons seulement, avec le professeur A. Jacquart, qu'il y a intrication entre biologique et relationnel pour qu'un trouble de ce type se manifeste.

${ }^{7}$ Rapport de l'INSERM, janvier 2006. Ce tri opéré sur des bases biogénétiques rappelle dangereusement les pires pratiques d'une idéologie que l'on pensait ne jamais devoir resurgir.
} 
l'amélioration des conditions de travail des ouvriers! Ceux-ci ne sont plus que des «objets jetables», des instruments à produire de l'argent pour les actionnaires.

Le fin du fin de la pensée entrepreneuriale pour augmenter la rentabilité est le licenciement : la «théorie du boucher» a remplacé la «théorie du boulanger» (HAMEL \& PRAHALAD, 2004). Il est plus simple, plus maîtrisable, plus «comptable», d'amputer l'entreprise d'une partie d'elle-même plutôt que de faire fermenter le levain qui est dans chaque sujet oeuvrant au développement de celle-ci. De quel côté de la rue est alors la «barbarie»?

Cette règle du tout évaluable et du plus rentable instaure donc des systèmes mettant aux commandes des comptables. Le comptable ne connaît pas le sujet. Or ces règles de rentabilité, par la mise en place de la Loi organique de finances (LOLF) qui régit depuis janvier 2006 le budget de l'Etat et des dépenses publiques, gouvernent également désormais le monde de l'action sociale. Face à ces exigences de rentabilité « à tout prix », chaque structure va, à court terme, se trouver devant l'alternative suivante : soit mettre les clés sous la porte, soit sélectionner le public qui lui permettra d'atteindre au plus près les objectifs à réaliser. Chaque structure sociale et d'insertion se positionnera donc comme une machine excluant les plus fragilisés, alors qu’elle devrait être à leur service.

De tels systèmes à l'intérieur d'un État font disparaître progressivement sa fonction de gouvernance et d'organisation de la vie sociale en ne lui laissant pour rôle que de garantir les frontières des îlots de dominance contre le reste de la société. En assurant la légalisation et la mise en œuvre des trois types de règles précédemment cités, l’État se réduit à n'être plus que le gendarme protecteur de ces îlots de dominance. C’est le «tout répressif» qui prédispose à la mort du sujet (BECK, 2003) dans un Etat devenu de plus en plus totalitaire. 


\section{L’injonction paradoxale «d'être sujet» au prix de «la mort du sujet»}

Pourtant, depuis sa naissance jusqu'à sa mort, le sujet est fortement incité à faire de sa vie «une œuvre» (KAUFMAN 2001, BOURRIAUD, 2003). Dans l’idéologie de la société postmoderne et néolibérale, cet individualisme, prôné à chacun par tous, est associé à l'exigence sacrosainte du «projet». Etre soi ne peut se définir que dans l'esprit de la construction d'un horizon personnel intégré à un semblant d’horizon global défini par l'Etat. Quand on entre par exemple dans une Mission Locale il n'est pas besoin d'attendre longtemps pour entendre un conseiller demander à un jeune: «mais quel est ton projet?». Pour le jeune qui est un sujet vivant une situation sociale globale, et non simplement un usager de la mission locale, cette question et les requisits qu'elle contient constituent une redoutable situation de double lien.

L'injonction paradoxale qui consiste à ordonner à une personne de faire en même temps une chose et son contraire (le doble bind de l'école de Palo-Alto) est psychiquement invivable. Demander à un jeune de dire «son projet», c'est-à-dire lui demander d'être «sujet désirant» dans un monde qui lui promet d'être licencié sans aucun motif ${ }^{8}$, ne peut qu'engendrer «la barbarie», autrement dit le déchaînement d'un désir qui n’a plus d’autre règle que lui-même. Mais n'est-ce pas là la seule manière d'éviter la folie? La folie ou la barbarie, voilà l'alternative qui resterait à ceux qui ne sont pas dans ces systèmes de «dominance internationale », sécrétion de la mondialisation post-moderne.

Pourtant cette «dominance mondiale» a besoin d'un reste du monde qui produise et qui consomme, et qui échappe donc à la folie et à la barbarie. N'est-ce pas alors la véritable commande faite au travail social? Maintenir du lien social, du sens, là où tout est rupture, exclusion, enfermement, non-

${ }^{8}$ C'était la disposition-phare du feu CPE. 
sens? Mais le travail social peut-il encore répondre à cette demande lorsqu'il va devoir lui aussi être évalué sur des critères de rentabilité, de productivité, à partir de la mise en place de la LOLF? Soumis à son tour aux règles de l’ingénieur, du médecin et du comptable, pourra-t-il maintenir «du sujet» dans des systèmes qui le nient?

\section{Travail social et politique du sujet: une gageure à tenir}

Mettre en oeuvre une «politique du sujet », c'est prendre à contrepied ces trois règles, qui ne peuvent prétendre rendre compte à elles seules de la réalité humaine. C’est travailler avec la complexité, avec les plis et replis de l'humain, ses ambiguïtés, ses contradictions, mais aussi avec ses désirs.

\section{Réintroduire la complexité en libérant la parole et l'imaginaire}

Pour nous, cela signifie en premier lieu libérer la parole du sujet. Libération du carcan de la rationalité scientifique, libération des espaces où cette parole peut se dire et s'entendre. Lorsqu'un jeune répond au conseiller de la mission locale que son projet est d'être médecin alors qu'il ne maîtrise pas les connaissances de base, la première réaction du conseiller est de lui montrer que cela est impossible (règle de l’ingénieur). Ce pragmatisme appuyé sur une prétendue logique des faits et de la pensée contribue à tuer encore plus du sujet dans le sujet. Un projet est toujours au début un projet imaginaire qui n’est pas régi par la logique mathématique, mais par la logique existentielle et émotionnelle du sujet. Cette logique-là doit avoir droit de cité pour tous et pas seulement pour quelques-uns. Si le conseiller accueille cette parole, recherche avec le jeune le désir qu'elle manifeste, il peut alors confronter ce désir à une réalité plus prosaïque et montrer à ce jeune que ce désir peut s’actualiser dans des métiers accessibles plus 
immédiatement, sans jamais fermer la porte à l'expression première du désir.

\section{Désir, co-construction et espaces de création}

Éduquer, former, accompagner la dimension de désir créatif du sujet, voilà la première mission que devrait se donner tout travailleur social, pour lui-même et pour son travail avec l'Autre. Mettant en pratique cette recommandation avec les jeunes qu'elle recevait dans une mission locale de la banlieue nord de Paris, une stagiaire de notre master ${ }^{9}$ fut étonnée des transformations intervenues dans le parcours de certains jeunes. Un jeune, qui était venu la voir à maintes reprises, se «permit» de dire, dès lors où elle libéra sa parole du carcan institué: «Tout ce que vous me proposez ne m'intéresse pas, moi c'est la musique qui m’intéresse». En travaillant à partir de cette parole, notre stagiaire co-construisit avec les jeunes un module de préparations aux métiers de la scène. Ces jeunes sont aujourd'hui sur des plateaux techniques de festivals ou de chanteurs. Libérer la parole créative, c’est travailler avec le désir propre du sujet et non pas avec celui qu'il est tenu de montrer quand, soumis à l'injonction de «dire son projet», il n’a d'autre solution pour échapper à une «mauvaise image supplémentaire de lui même» que d'en définir un, n’importe lequel (BIARNÈS, 1993, BIARNÈS \& AZOULAY, 1995).

Cette reconnaissance du sujet et de sa parole propre implique que l'acteur social fasse «avec» le sujet et non «pour» lui. «Faire avec» c'est aussi mettre en œuvre la seconde libération de la parole, celle qui consiste à la faire advenir en tout lieu. L'altermondialisme, les coordinations de grévistes ou d'étudiants, les manifestations des infirmières représentent avant tout cette reprise sur toutes les scènes de la vie sociale du pouvoir de la parole libérée, dont le monde a besoin aujourd’hui comme contre pouvoir

\footnotetext{
9 Master 2 professionnel « Ingénierie de programme d’insertion », Université de Paris-13.
} 
à la parole dominante. Mais les moindres petits espaces sont aussi des contre-pouvoirs. Nous l'avons vu lorsque nous préconisions à un préfet d'entendre directement la parole des bénéficiaires du RMI, mais cela est vrai pour chaque usager d'un service médico-social. Combien de citoyens n’osent pas interroger le discours du médecin, tellement ce dernier est péremptoire et totalitaire, que le médecin joue ou non de cette toutepuissance. Depuis cinquante ans, on démontre aux parents qu'ils ne savent pas éduquer, qu'ils doivent faire appel à des professionnels qui eux ont été formés et qui possèdent donc l'«expertise» et la «compétence». Le résultat en est que les parents sont aujourd'hui accusés de démission par l'Etat ou ses représentants. L’acteur social n'échappe pas à ce discours dominant qu'il colporte et l'empêche de laisser la place à l'émergence du discours de l'usager.

\section{Etre «centré sur la personne» et non pas «la mettre au centre de...»}

Pour se «décentrer» de ces habitus, pour être capable de promouvoir dans son travail ces espaces de paroles, nous préconisons à l'acteur social un exercice à mener chaque jour: interroger pour les éliminer certains concepts «pré-fabriqués» qui impliquent des postures professionnelles propres à exclure la parole du sujet, donc le sujet lui-même. «Mettre la personne (élève ou usager) au centre du dispositif» éducatif ou social, engage l'acteur social à penser et à faire "pour» le sujet et donc à le réifier (parole de l’ingénieur). Ce sont des objets que l'on place quelque part, non des personnes. Dans un dispositif est totalement construit autour de la personne, quel est l'espace de liberté de celle-ci, sinon de le fuir ? Un dispositif conçu «autour» de la personne est sur une logique de négation de la personne en tant que sujet. En revanche, «être centré sur la personne» engage le travail 
social dans une tout autre dimension, celle de la reconnaissance de l'autre comme sujet désirant, parlant et co-agissant.

\section{La personne n'est pas EN difficulté, elle rencontre des difficultés}

De même nous préconisons d'éliminer le vocable répandu de «personne en difficulté» pour le remplacer par «personne rencontrant des difficultés». La première formulation engage l'enseignant ou l'acteur social à concevoir que la difficulté est dans le sujet et donc à en rechercher les causes dans la personne comme si elles étaient constitutivement ancrées dans sa «nature» (parole du médecin). Je montre à mes étudiants deux séquences filmées auprès de jeunes en institutions psychiatriques. L’un est capable de dire, plus vite qu'un ordinateur, le jour, le mois ou l'année qui présente telle ou telle particularité en fonction de la question posée. Le second est capable de rejouer au piano, après seulement trois auditions, une mélodie complexe spécialement écrite afin de s'assurer qu'il ne l'ait jamais entendue. Si nous prenions comme référence les capacités de ces deux jeunes, aucun d'entre nous n'échapperait à un étiquetage catastrophique de son quotient intellectuel: nous serions alors étiquetés «en difficulté» et serions soumis à des techniques «rééducatives» pour réparer nos manques. Cet exemple montre bien que «la difficulté» n’est pas dans notre «nature» mais existe dans le conflit qui naît entre la demande sociale et les possibilités de réponse que nous lui apportons. Autrement dit la posture du professionnel de l'action sociale ou de l'enseignant est de travailler avec le sujet à transformer cette relation dont les résultats ne sont pas satisfaisants de son point de vue et non pas du nôtre. Pour cela, il devient indispensable à l'acteur social de faire émerger chez l'Autre ses potentiels et non de comptabiliser ses manques (parole du comptable) afin qu'il puisse être cocréateur d'une nouvelle relation avec son environnement. 


\section{Remettre l'exilé de lui-mêe en lui-même, la biographisation réciproque et le sens}

Nous disions ci-dessus que «la mort» du sujet venait aussi de la segmentation et de la dé-localisation qu’on prétend appliquer à ce qu'il est. Réunifier le sujet en un tout, le re-situer dans un espace social dont il peut avoir les outils de compréhension, voilà encore un aspect de ce que devrait être une politique du sujet dans le travail social. Le travail de biographisation de leur trajectoire paraît aujourd'hui incontournable pour ces sujets parcellisés et dé-territorialisés (DELORY- MOMBERGER, 2003, 2005). Sans un travail de conscientisation et d'appropriation de son parcours, comment être sujet de son histoire? La biographisation tend à remettre l'exilé de lui-même en lui-même. Dans l'action sociale, nous savons bien que les personnes exclues du monde du travail se désocialisent, perdent les repères de vie au quotidien. Si l'acteur social ne prend pas le temps de reconstruire avec la personne une «consistance» de sa trajectoire personnelle, il y a de grands risques pour que l'usager d'un dispositif social ne parvienne jamais à investir une position de sujet. Mais le peut-il si l'intervention de l'acteur social se réduit à solliciter de lui «un récit de sa vie»? Le travail de biographisation auquel nous appelons devrait prendre la forme de la «biographisation réciproque» (BIARNÈS, 2006), c'est-à-dire d'un dévoilement réciproque de la personne et de l'acteur social au croisement d'un «vivre ensemble», où l'on a besoin de dire son passé pour faire comprendre à l'autre le présent et co-construire avec lui l'avenir. Livrer un récit de vie engendre immédiatement une dynamique interprétative de la part de l'acteur social qui renvoie l'usager à la position d'objet. La «biographisation réciproque» prend le sens d'un partage, celui de se comprendre l'un l'autre pour réussir ensemble un acte de construction de soi. Elle présente la double efficacité de «re-situer» le sujet dans un contexte et de l'amener à re-composer un sens à sa trajectoire personnelle. 


\section{Accompagner le double mouvement de «l'insertion/désinsertion»:}

Une politique du sujet dans le travail social ne peut se concevoir sans faire référence au double mouvement d'«insertion/désinsertion» qui constitue le principe dynamique du sujet en tant qu'il est un processus toujours à l'oeuvre. C'est ce que nous nommons le jeu narcissique du sujet. En effet quand le sujet tente de réduire le conflit entre les identifications qu'il devrait construire «en cheminant vers demain» et sa propre histoire telle qu'elle est à ce jour, il le fait de manière à garantir l'amour qu'il a de lui-même. C'est ce que nous pouvons faire entrer dans ce que certains auteurs appellent «le narcissisme primaire», et ce que Freud définit comme «le retour de la libido sur des objets intériorisés» (1912). La souffrance de devoir abandonner un certain nombre d'identifications secondes doit trouver sa compensation immédiate dans la continuation à s'aimer tel que l'on est. C'est bien ce que nous montrent des textes de jeunes de 16 à 25 ans rencontrant des difficultés d'insertion sociale et professionnelle et recueillis lors d'un atelier d'écriture. Nous citons l'exemple d'un texte écrit sur le thème de la personne que l'on aimerait rencontrer:

Je ne sais pas quoi dire, à vous qui me ressemblez comme deux gouttes d'eau. Sans vous chercher, je vous ai trouvé, j'ai remarqué que vous aviez les mêmes habitudes que moi, vous regardant courir, manger, fumer. Je ne sais pas comment vous décrire, mais vous êtes exactement la réplique de moi-même.

Lorsque le sujet en reste à ce miroir de lui-même, il n'est pas étonnant que l'environnement le «voit» limité dans ses possibilités intellectuelles, «sympathique» sûrement, en souffrance toujours. Il faudra toute la perspicacité de l'acteur social pour «entendre», au-delà du manifeste, les désirs profonds du «sujet» et pour «retourner» la problématique de la souffrance à grandir en la jouissance de grandir. 
L’importance devient ici «l'accompagnement» du sujet. Si ce dernier n’a pas autour de lui des adultes qui peuvent être des repères indispensables pour, à la fois, le «nourrir» et lui «permettre» de négocier ses conflits entre les modèles qui lui sont proposés, il adoptera la solution de la moindre souffrance, celle de ne plus bouger. Apprendre, grandir, se transformer ne se fait jamais dans «le silence du monde» comme disait Albert Camus. Personne ne peut apprendre seul, grandir seul, se construire ou se reconstruire seul. C’est toujours un travail en interaction, en interactivité avec les autres.

\section{Une «politique du sujet» dans le travail social est-elle encore possible?}

La mondialisation, nous l'avons dit, met de plus en plus «les comptables» aux commandes. Ce mouvement s'est amplifié avec la décentralisation parce que les collectivités territoriales ont été prises dans une contradiction qu'elles ont essayé de résoudre par la voie la plus simple. D’une part, elle devenaient les «financeurs» de l'action sociale et à ce titre devaient avoir des exigences. D'autre part elles ne «savaient pas faire», c'est-à-dire monter, organiser, animer et évaluer des politiques sociales puisque cela était auparavant dévolu à l'Etat. La mondialisation et la décentralisation firent passer les politiques sociales d'une obligation de moyens à une obligation de résultats.

Dans une logique mathématique et comptable, pour arriver à satisfaire une «obligation de résultats», il faut posséder des instruments de pilotage fiables. Autrement dit, il ne suffit pas d'effectuer une évaluation finale pour s’apercevoir des écarts entre le projet et la réalisation, il faut avoir des indicateurs de veille tout au long de l'action afin d'être averti en temps réel du moindre écart entre les prévisions et les réalisations. Les Missions Locales se sont ainsi dotées d’un logiciel, «Parcours 3», pouvant 
fournir à chaque instant un diagnostic de la situation. Pour que ce logiciel puisse être l'outil de pilotage sensible que souhaitaient les décideurs, il a été construit sur une standardisation des items à renseigner. On est bien loin de la diversité des publics accueillis. Comme, de plus, les subventions aux Missions Locales se basent sur un premier indicateur qui est celui du nombre de jeunes accueillis, l'on comprend bien que l'accueil peut devenir une simple salle d'enregistrement de données, et que le risque est grand que le conseiller d'accueil renseigne directement les items du logiciel, les yeux fixés sur l'écran plutôt que sur la personne qui lui parle.

Cette vision «productiviste» de l'action sociale, s'est également concrétisée par la Loi organique des lois de finances qui «découpe» le social en critères quantitativement repérables et donc évaluables. Toute l'action sociale est aujourd'hui considérée au même titre qu'une activité de production de biens. Elle doit se fonder sur un projet de résultats en fonction des moyens mis à sa disposition et doit rendre compte périodiquement de l'avancée de l'action et du résultat final. Les moyens seront calculés en fonctions des résultats obtenus. Bien évidemment les critères quantitatifs sont ceux du financeurs et non ceux qui peuvent prendre en compte le sujet. Dans un programme P.A.QU.E ${ }^{10}$ qui avait pour objectif le placer en emploi ou en formation qualifiante d'au moins $70 \%$ des 100.000 jeunes sortant du système éducatif sans maîtriser les connaissances de bases, une jeune femme de 20 ans avait comme projet personnel d'apprendre à lire pour suivre et aider son enfant qui allait entrer au cours préparatoire. Sa motivation était tellement forte qu'elle y réussit. Sur les critères du commanditaire, elle fut comptabilisée comme «échec»! Pourtant pour elle, la réussite du programme aurait été mesurable à l'aide qu'elle a pu apporter à son enfant. Les critères quantitatifs ne peuvent jamais rendre compte du réel humain.

${ }^{10}$ Préparation Active à la Qualification et à l'Emploi, programme initié par Mme. Aubry, alors ministre du travail 
Dans de telles conditions, une «politique du sujet est-elle encore possible? En travaillant avec des conseillers de deux Missions Locales au titre d'un programme EQUAL sur l'accompagnement, nous avons pu construire ce que pouvait être un accompagnement prenant en compte en même temps que la dimension quantitative la dimension qualitative du rapport à la personne en tant que sujet. Ainsi, chaque étape de la chaîne de l'accompagnement commence non pas par la préconisation de «la» bonne démarche à suivre, mais «des bonnes questions» à se poser en fonction de ce que nous avons appelé «les fondamentaux de l'accompagnement» pour que la personne reste sujet de son parcours. ${ }^{11}$

Les critères quantitatifs, légitimes pour le financeur, ne doivent pas être les seuls à rendre compte de l'action sociale, sinon toutes les dérives sont possibles. D’une part les acteurs focalisés sur ces critères excluront de facto l'usager d'une place de sujet pour le placer en situation d'objet de leur propre action. Eux-mêmes seront dans l'impossibilité d'analyser le sens de cette action puisqu'elle se limitera à essayer de répondre au plus près à l'injonction créée par les indicateurs. Comme les usagers, les acteurs sociaux seront instrumentalisés et donc placés en situation d'assujettissement à la politique sociale. D'autre part, toute structure devra, pour sa survie et pour ne pas mettre ses personnels au chômage et donc en situation d’être les nouveaux bénéficiaires de l'action sociale, «trier» son public. En fonction des objectifs que se fixera la structure, elle devra sélectionner les publics qui devraient répondre au mieux et au plus vite à ses objectifs. On en vient de nouveau à la constitution d'un système fonctionnant sur lui-même, et donc à la fabrication d'une machine excluante à partir d'une structure faite pour l'insertion.

Il est en conséquence urgent que les acteurs sociaux se soucient de construire leurs propres instruments d'évaluation de leur travail, instruments

\footnotetext{
${ }^{11}$ Les conclusions de ce programme EQUAL ne sont pas encore publiées mais peuvent être consultés à Paris-13 à la bibliothèque des Sciences Humaines.
} 
d'évaluation qualitative et non quantitative. En ce sens nous avons travaillé avec les encadrants de quatorze chantiers d'insertion sur tout un département. Nous avons pris le prétexte de co-construire un «carnet de bord » pouvant servir à la fois à l'usager et à l'encadrant. Les rubriques de ce carnet de bord sont somme toute classiques: savoirs, savoir-faire, savoirêtre. Pour chacune de ces trois rubriques nous avons créé des «items ». Chacun d'eux sont évaluables de un à cinque, le un marquant l'absence de maîtrise de l'item, le cinque à l'inverse sa bonne maîtrise. Ces items sont à remplir à la fin des premiers, troisième et sixième mois. L'encadrant rempli un carnet «Monsieur X » et séparément, sans contact, Monsieur X rempli son propre carnet. La rencontre entre l'encadrant et Monsieur X a pour objet la comparaison des deux évaluations et l'analyse des écarts et des correspondances dans les notations. Le but ultime n’est pas de «négocier» une évaluation commune, amis de bien comprendre les raisons qu'ont eues chacun des deux évaluateurs pour noter comme ils l'on fait.

Nous sommes là dans une vraie dynamique du sujet, autant du côté de l'usager que de celui de l'encadrant. L'évaluation établie par l'un et par l'autre et sa légitimité sont reconnues comme telles (prise en compte de la parole et émergence de cette parole en un lieu jusque là interdit, celui de sa propre évaluation), mais chacun a l'obligation de faire comprendre à l'autre cette légitimité. Pour ce faire, chacun a besoin de se «dévoiler» (biographisation réciproque), chacun a besoin d'expliciter ses critères au regard de situations vécues en commun (re-territorialisation de la personne) et chacun doit essayer de rester cohérent (déparcellisation de la personne dans ces différents parcours). Enfin, avec cette technique d'évaluation, l'encadrant est à même d'avoir une analyse qualitative de son action avec chaque personne qu'il accompagne et de pouvoir la confronter au critères quantitatifs de la LOLF.

Une politique du sujet dans l'action sociale est pour nous encore possible si les structures et les acteurs sociaux sont eux-mêmes 
accompagnés dans une analyse en continue de leur action, ce que nous appelons le «tiers analysant»(DAHAN, 2005). Maintenir et développer cette politique du sujet en éducation et dans l'action sociale est l'un des moyens de ne pas conforter un monde clivé entre dominance et barbarie, c'est faire en sorte que le sens de l'humain ne disparaisse pas pour la seule raison qu'il n'est pas rentable, et c'est à tout le moins empêcher que les modes de dominance qu'installent les règles de l'ingénieur, du médecin et du comptable, ne tuent «l'être-sujet». Nous pourrions tous, enseignants et acteurs sociaux en priorité, faire nôtre ce principe rappelé par Raoul Vaneigem (VANEIGEM, 2004, p.102), «l'humain prime le nombre».

Si faire vivre une politique du sujet à toujours été une question d'éthique, c'est aussi aujourd'hui une question de démocratie.

\section{Bibliographie}

Beck, U. (2003). Pouvoir et contre pouvoir à l'ère de la mondialisation. Paris: Aubier.

Benasayag, M. (2004). Le Mythe de l’individu. Paris: Éd. La découverte.

Biarnès, J. (1993). Le Projet assassin d'avenir. acte du colloque CREAI, Dijon.

Biarnès, J. (1999). Universalité, diversité, sujet dans l'espace pédagogique. Paris: L’Harmattan.

Biarnès, J. (2006).(à paraître): «Maintenant je ne suis plus invisible, chantiers d'insertion et politique sociale», in $\mathrm{n}^{\circ}$ spécial OSP Insertion, biographisation, éducation. coord. Biarnès J./ DeloryMomberger Ch.

Biarnès, J. \& Azoulay A. (1995). «Le bilan intégré à l'acte formatif», in Les jeunes, l’insertion, l'emploi. Charlot B. \& Glasman D. (coord.), Paris: PUF. 
Bourriaud, N. (2003). Formes de vie, L'art moderne et l'invention de soi. Paris: Denoël.

Dahan, L. (2005). Le Tiers analysant. Mémoire de DEA, CNAM, chaire du Travail Social.

Delory-Momberger, Ch. (2003). Biographie et éducation. Figures de l'individu-projet. Paris: Anthropos.

biographique en éducation. Paris: Anthropos.

(2005). Histoire de vie et recherche

Freud, S. (1912). Totem et tabou. Paris: PUF.

Habermas, J. (1987). Théorie de l’agir communicationnel. Paris: Fayard.

Hamel, G. \& Prahalad (2004). Competing for the future. HBR.

Kauffman, J.C. (2001). Ego. Pour une sociologie de l'individu. Paris: Nathan.

Mongin, O. (2005). La condition urbaine. La ville à l'heure de la mondialisation. Paris: Seuil.

Morin, E. (1992). Introduction à la pensée complexe. Paris: ESF.

Vaneigem, R. (2004). Pour l'abolition de la société marchande, pour une société vivante». Paris: Payot \& Rivages. 


\section{Autor:}

\section{Jean Biarnès}

Professeur à l’Université Paris 13/Nord

Centre interuniversitaire de recherche EXPERICE (Paris 13-Paris 8)

jhbiarnes@aol.com

\section{Como citar este artigo:}

BIARNES, Jean. Mondialisation, travail social et politique du sujet. Revista ACOALFAplp: Acolhendo a Alfabetização nos Países de Língua portuguesa, São Paulo, ano 2, n. 4, 2008. Disponível em: $<$ http://www.mocambras.org > e ou <http://www.acoalfaplp.org>. Publicado em: março 2008.

Recebido em setembro de 2006./ Aprovado em dezembro de 2006. 